\title{
S2RSLDB: a comprehensive manually curated, internet-accessible database of the sigma-2 receptor selective ligands
}

\author{
Giovanni Nastasi, Carla Miceli, Valeria Pittalà, Maria N. Modica, Orazio Prezzavento, Giuseppe Romeo, \\ Antonio Rescifina, Agostino Marrazzo and Emanuele Amata* ${ }^{*}$
}

\begin{abstract}
Background: Sigma ( $\sigma)$ receptors are accepted as a particular receptor class consisting of two subtypes: sigma-1 $\left(\sigma_{1}\right)$ and sigma-2 $\left(\sigma_{2}\right)$. The two receptor subtypes have specific drug actions, pharmacological profiles and molecular characteristics. The $\sigma_{2}$ receptor is overexpressed in several tumor cell lines, and its ligands are currently under investigation for their role in tumor diagnosis and treatment. The $\sigma_{2}$ receptor structure has not been disclosed, and researchers rely on $\sigma_{2}$ receptor radioligand binding assay to understand the receptor's pharmacological behavior and design new lead compounds.
\end{abstract}

Description: Here we present the sigma-2 Receptor Selective Ligands Database (S2RSLDB) a manually curated database of the $\sigma_{2}$ receptor selective ligands containing more than 650 compounds. The database is built with chemical structure information, radioligand binding affinity data, computed physicochemical properties, and experimental radioligand binding procedures. The S2RSLDB is freely available online without account login and having a powerful search engine the user may build complex queries, sort tabulated results, generate color coded 2D and 3D graphs and download the data for additional screening.

Conclusion: The collection here reported is extremely useful for the development of new ligands endowed of $\sigma_{2}$ receptor affinity, selectivity, and appropriate physicochemical properties. The database will be updated yearly and in the near future, an online submission form will be available to help with keeping the database widely spread in the research community and continually updated. The database is available at http://www.researchdsf.unict.it/S2RSLDB.

Keywords: Sigma receptor, Sigma-2 receptor, Online ligand database, Structure search, S2RSLDB, 2D plot, 3D plot, Drug design, Central nervous system multiparameter optimization, Lipinski's rule of five

\section{Background}

Sigma $(\sigma)$ receptors are accepted as a particular receptor class consisting of two subtypes: sigma-1 $\left(\sigma_{1}\right)$ and sigma-2 $\left(\sigma_{2}\right)$. They are distinguished by molecular weight (MW), drug actions, pharmacological profiles and molecular characteristics $[1,2]$. The $\sigma_{1}$ receptor has a MW of $25.3 \mathrm{kDa}$ and was first cloned from guinea pig liver (UniProtID Q60492, Gene names SIGMAR1, CHEMBL4153) in 1996 [3, 4], and afterwards from human placental

\footnotetext{
*Correspondence: eamata@unict.it

Department of Drug Sciences, Medicinal Chemistry Section,

University of Catania, Viale A. Doria 6, 95125 Catania, Italy
}

choriocarcinoma cell (UniProtID Q99720, Gene names SIGMAR1, CHEMBL287) [5]. In addition, the $\sigma_{1}$ receptor was also cloned by other organisms, like mouse (UniProtID O55242, CHEMBL3465), rat (UniProtID Q9R0C9, CHEMBL3602), brushtail possum (UniProtID Q5PXE2), ermine (UniProtID Q5PXE3), bovine (UniProtID Q58DH7), chicken (UniProtID Q5ZL84), and zebrafish (UniProtID Q7ZWG9). Recently the crystal structures of the human $\sigma_{1}$ receptor in complex with two ligands, has been reported (PDB ID 5HK1 and 5HK2) [3, 6].

The $\sigma_{2}$ receptor has not yet been cloned or crystallized and the knowledge about this receptor has mostly been generated through in vitro receptor radioligand 
binding studies $[7,8]$. As reported the $\sigma_{2}$ receptor has a MW between 18 and $21 \mathrm{kDa}[9,10]$. It has been postulated that the $\sigma_{2}$ receptor binding site may be located in the progesterone receptor membrane component 1 (PGRMC1), even if its MW (25 kDa) is different from that of $\sigma_{2}$ receptor $[10,11]$.

The $\sigma_{1}$ receptor is involved in aging and various diseases, like schizophrenia, depression, Alzheimer's disease and ischemia. The $\sigma_{1}$ receptor agonists have showed neuroprotective, anti-amnestic and antidepressant effects [12-14]. Conversely, $\sigma_{1}$ receptor antagonists are considered antiproliferative, antiangiogenic and to have modulatory effects on opioid analgesia [15-17]. Some studies suggested that $\sigma_{1}$ receptor is involved in modulating the synthesis and release of dopamine and also to act as a molecular chaperone at the mitochondrion-associated endoplasmic reticulum membrane (MAM) where it regulates calcium signaling between the two organelles [4, 18-20].

Despite the lack of structural information, the $\sigma_{2}$ receptor has gained remarkable attention due to its involvement in several human diseases, including but not limited to depression, anxiety and cancer diagnosis and treatment [21-23]. The $\sigma_{2}$ receptor ligands determine tumor cell death through apoptotic and non-apoptotic pathways, although their mechanisms of action have not been fully elucidated [24, 25]. In addition, the overexpression of $\sigma_{2}$ receptor in several tumor cell lines is noteworthy [26-28]. The $\sigma_{2}$ receptor is expressed about tenfold more in proliferating tumor cells compared with quiescent tumor cells, keeping the $\sigma_{2}$ receptor ligands highly indicate for ligand-targeted cancer therapeutic strategies and as imaging agents [23, 29-32]. This peculiarity has been used for the development of $\sigma_{2}$ receptor selective ligands as Positron Emission Tomography (PET) imaging tools. $\left[{ }^{18} \mathrm{~F}\right] \mathrm{ISO}-1$, a promising PET ligand targeting $\sigma_{2}$ receptor, has been evaluated in clinical trial for the assessment of cellular proliferation in tumors by PET and three additional phase I clinical trials on this compound are actually ongoing [33-36]. These differences in the pharmacological profiles of the $\sigma$ receptor subtypes, prompt to a continue research of ligands that selectively target each of them. However, whilst several ligands selectively bind to the $\sigma_{1}$ receptor or indistinctly to the two receptor subtypes, the development of compound endowed with high selectivity for the $\sigma_{2}$ receptor has been challenging and in some cases occurred through an accidental discovery [23].

Due to the lack of structural information about the $\sigma_{2}$ receptor and its growing implication in cancer diagnosis and treatment, a thorough and in-depth collection of the selective $\sigma_{2}$ receptor ligands could result in a helpful tool for drug discovery. Herein, an online ligand database named sigma-2 Receptor Selective Ligands Database (S2RSLDB) based on 2D structural information, computed physicochemical properties, pharmacological properties together with the experimental procedure protocols, retrieved from the literature, has been built and resulted in more than 650 compounds. The database contains all the ligands that selectively bind the $\sigma_{2}$ receptor (i.e. $\left.K_{\mathrm{i}} \sigma_{1} / \sigma_{2}>1\right)$. The S2RSLDB is freely available online without account login and having a powerful search engine the user may build complex queries, sort tabulated results, generate color coded 2D and 3D graphs and download the data for additional offline screening.

The collection here reported is extremely useful for the development of ligands endowed of $\sigma_{2}$ receptor affinity, selectivity, and appropriate physicochemical properties. To the best of our knowledge, there is not any online database reporting such complete compounds map for this receptor. Moreover, in most cases these do not allow a comparison between the compound's features and a complete and correct set of compounds is difficult to be returned. The database will be updated yearly and in the near future, an online submission form will be available to help with keeping the database widely spread in the research community and continually updated.

\section{Description and utility}

Compound information was manually retrieved from the literature, including journal articles and patents, which were selected using major databases like Pubmed [37], SciFinder [38], and Google. The Binding Database [39], ChEMBL (v21) [40], PubChem [41], PDBbinding [42], ChemSpider [43], as well as other online ligand databases, were also checked for completeness. For each literature source, a curator manually constructed the 2D chemical structures using Marvin Sketch (v14.9.1.0) [44] and converted them into SMILES strings using JChem for Excel (v14.9.100.809) [44]. For few publications, the Optical Structure Recognition Application (OSRA) software (v2.0.1) was employed to generate SMILES [45]. A SMILES file was generated and then converted them into structures using JChem for excel (v14.9.100.809) [44]. A second curator visually inspected for common structure mistakes. Once a full list of compounds was available, compound structures were verified in SciFinder [38], patent and journal articles by SMILES or 2D structure visual inspection. Finally, Open Babel (v2016-01) was used to create InChI strings that in turn were used to remove duplicates from the S2RSLDB [46].

Other information taken from the literature includes the radioligand binding affinity values $\left(K_{\mathrm{i}}\right.$ or $\mathrm{IC}_{50}$ expressed in $\mathrm{nM}$ or otherwise converted in $\mathrm{nM}$ ), the experimental procedure protocols [47], the reference article compound key and/or preferred name (e.g. 


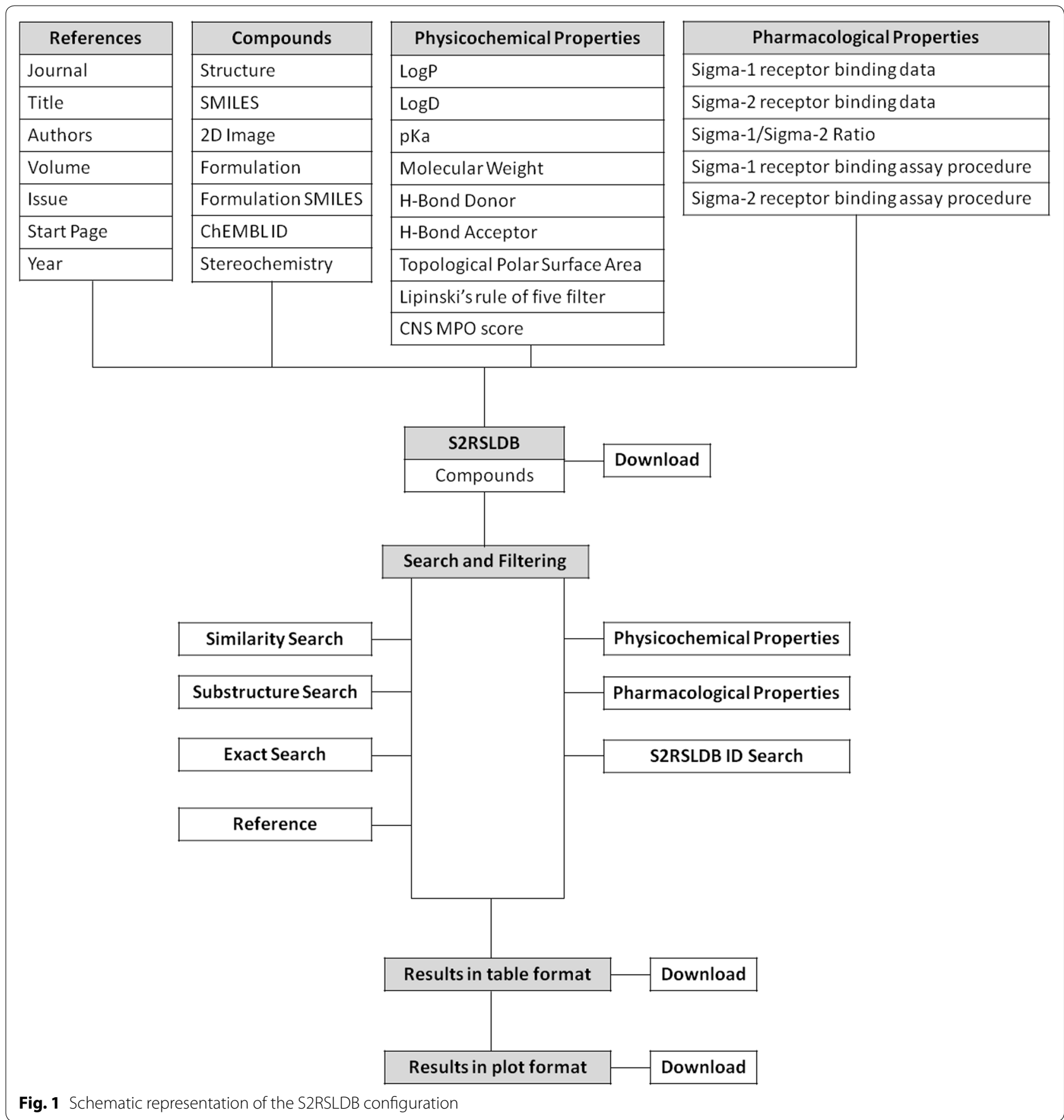

CM-361; 9f), and the compound formulation (free base or salt) used for the displacement binding assay. Those compounds presenting numerous binding affinity values, resulted from different experimental conditions, have been associated with multiple experimental data. Indeed, for several compounds, significant variations in binding assay output values have been encountered and for others an inversion in the selectivity ratio $\left(K_{\mathrm{i}} \sigma_{1} / \sigma_{2}<1\right)$ has been observed.

S2RSLDB is available online at http://www.researchdsf.unict.it/S2RSLDB. The database is implemented in MySQL (v5.1.73) with Apache (v2.4.20) as the web server. For chemical calculation and structure drawing Open Babel (v2016-01), Pybel (v2.3.1) and JSME Applet 
(v2015-12-06) are incorporated [46, 48, 49]. Other functions are made available with Python scripts and background program. The data are stored in MySQL (v5.1.73) database. The website is built in HTML, JavaScript, CSS and PHP. Compound images have been generated through Indigo Toolkit (v1.2.1) [50]. The types of data stored and the database structure are illustrated in Fig. 1.

The S2RSLDB has a powerful and intuitive web interface. The search page is divided into five sections: structure, computed physicochemical properties, pharmacological properties, reference search, and S2RSLDBID. Structure search may be performed by either drawing a molecule using the JSME Molecular Editor or entering a SMILES string as query input. Within the structure search, similarity, substructure, and exact search can be run. Similarity search employs the FP2 fingerprint, substructure search is performed via SMARTS while exact search is done through InChI strings correlation [51-53]. By default, the website is set to perform similarity search with a Tanimoto coefficient cutoff of zero [51]. With this setting, all the compounds in the database are returned to the query, and the result webpage will give a tabulation of the compounds sorted by Tanimoto coefficient. All the aforementioned functionalities are done via Pybel [48].
Physicochemical properties search contains selected molecular properties which were calculated for all compounds using ChemAxon's calculator cxcalc (v6.1.3) [44]. These descriptors include MW, octanol-water partition coefficient ( $\log \mathrm{P}), \mathrm{H}$-bond donors (HBD), H-bond acceptors (HBA), which allow the creation of the Lipinski's rule of five filter [54]. A Lipinski's rule of five filter checkbox has been incorporated for helping the end user in automatically set the filter cutoff. Other descriptors include $\log \mathrm{D}[\mathrm{pH}$ 7.4], topological polar surface area (TPSA), atom count, and $\mathrm{p} K_{\mathrm{aH}}$ (calculated for the most basic center). A histogram distribution of these computed physicochemical properties for the compounds in the database is presented in Fig. 2. For alignment of the key druglike attributes, the central nervous system multiparameter optimization (CNS MPO) score has been calculated for each compound and a CNS MPO score box has been included in the search page utility interface [55]. This score (0-6 range) is the sum of a set of six normalized ( 0 to 1 range) physicochemical parameters: $\log \mathrm{P}, \log \mathrm{D}, \mathrm{MW}, \mathrm{TPSA}, \mathrm{HBD}$, and $\mathrm{pK}_{\mathrm{aH}}$ and may help the user to predict each compound's likelihood of CNS activity and overall better druglike properties [55, 56]. Compounds having a CNS MPO $\geq 4$ show better druglike

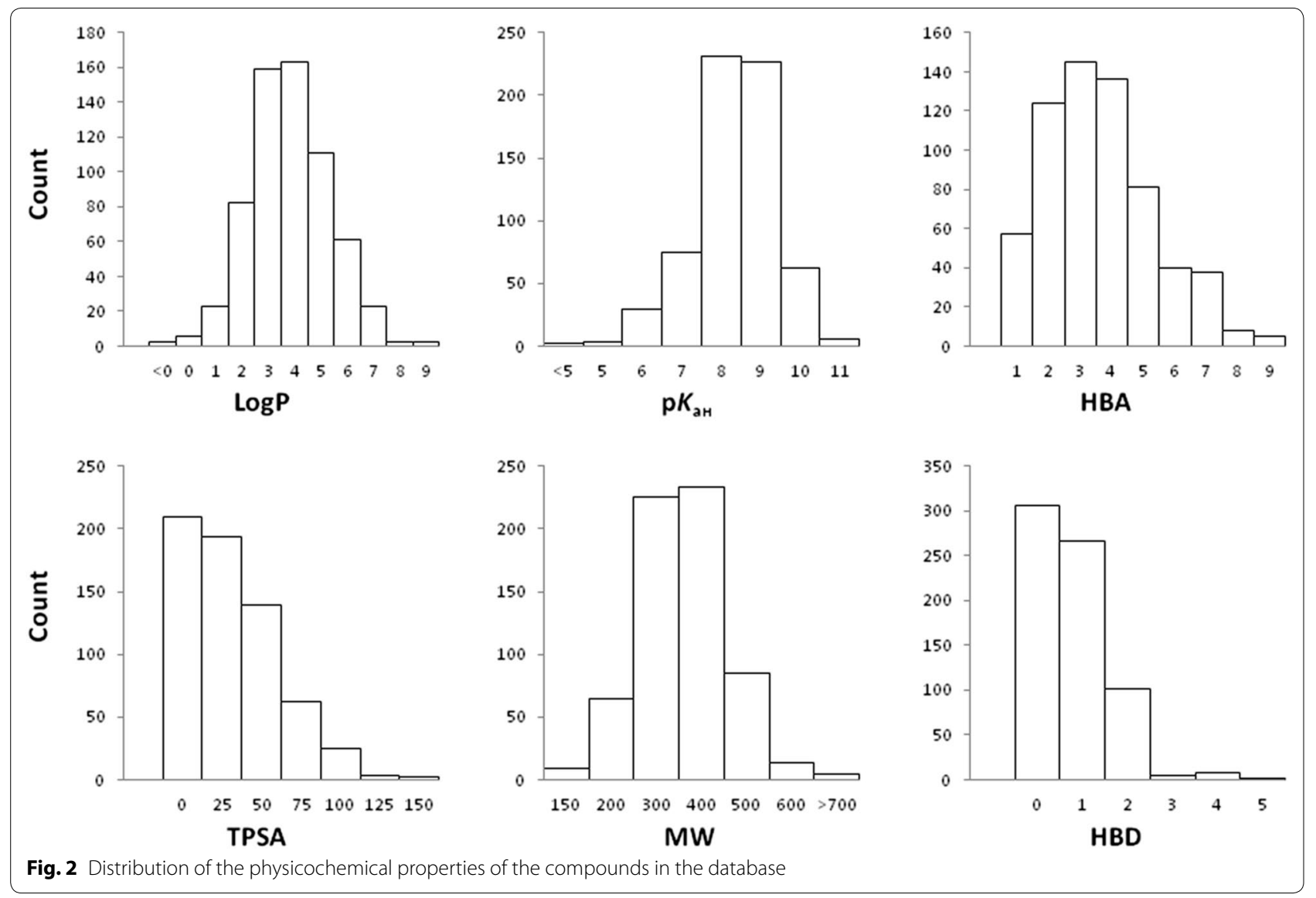


properties and are predicted to be centrally acting. [55, 56]. Furthermore, by activating the CNS MPO attributes input checkbox the six physicochemical properties boxes composing the score, will be returned in the normalized fashion according to the CNS MPO algorithms. For each computed physicochemical property, cutoff or range can be specified by changing the number in the search webpage boxes. In Fig. 3a, Lipinski's rule of five filter was applied together with a substructure search of 1,2,3,4-tetrahydroisoquinoline and this returned 67 hit compounds subjected to the rules. Once the search function is launched, matching queries are returned on a result webpage in tabulated format displaying main pharmacological and computed physicochemical properties together with the CNS MPO score as well as the 2D image of the compounds (Fig. 3b). On condition that the CNS MPO input checkbox has been activated, the result webpage will show the six computed physicochemical
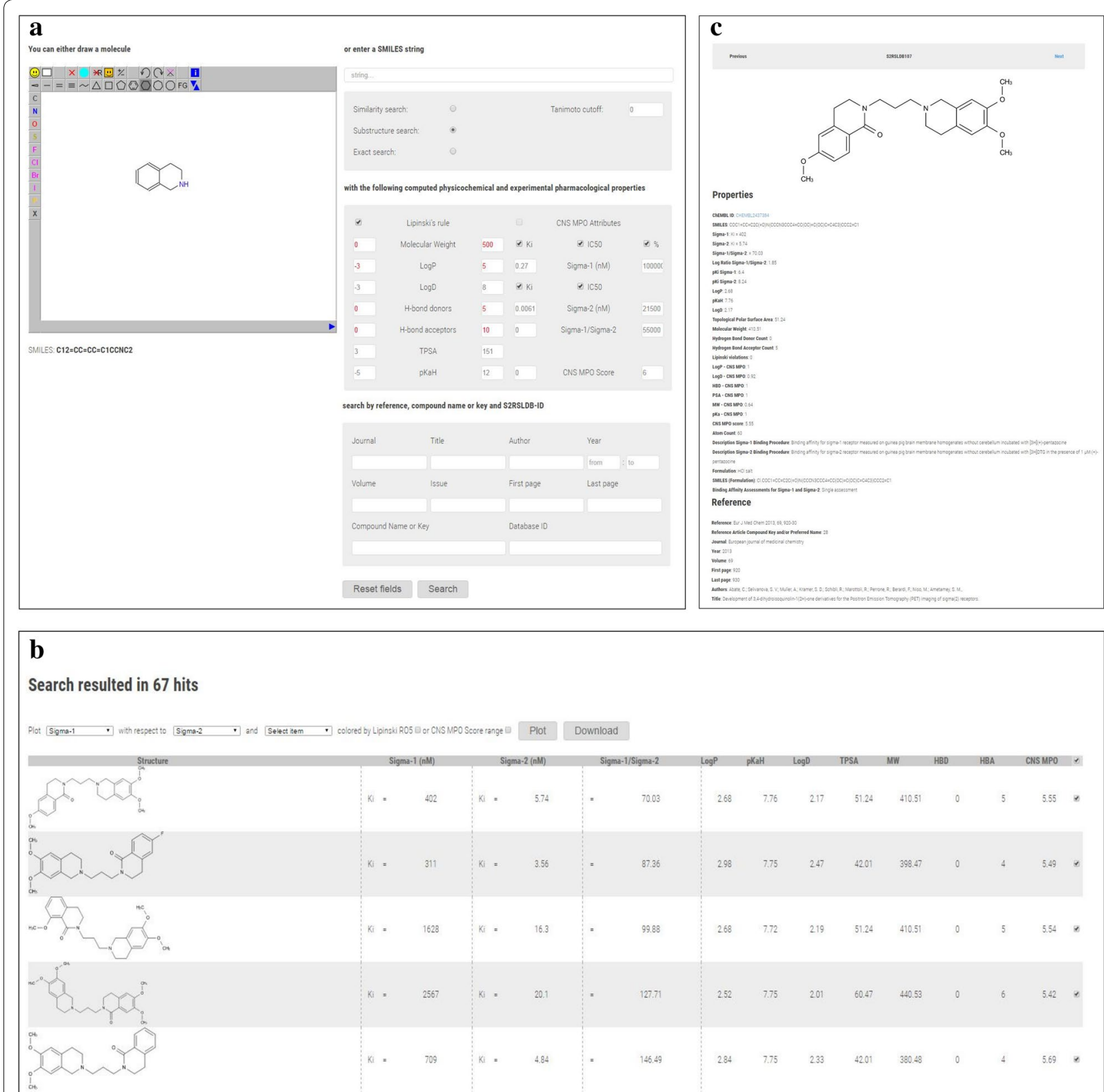

Fig. 3 Screenshots of the compound search page set with 1,2,3,4-tetrahydroisoquinoline substructure search and Lipinski's rule of five filter (a), tabulated results page $(\mathbf{b})$, and compound summary page $(\mathbf{c})$ 

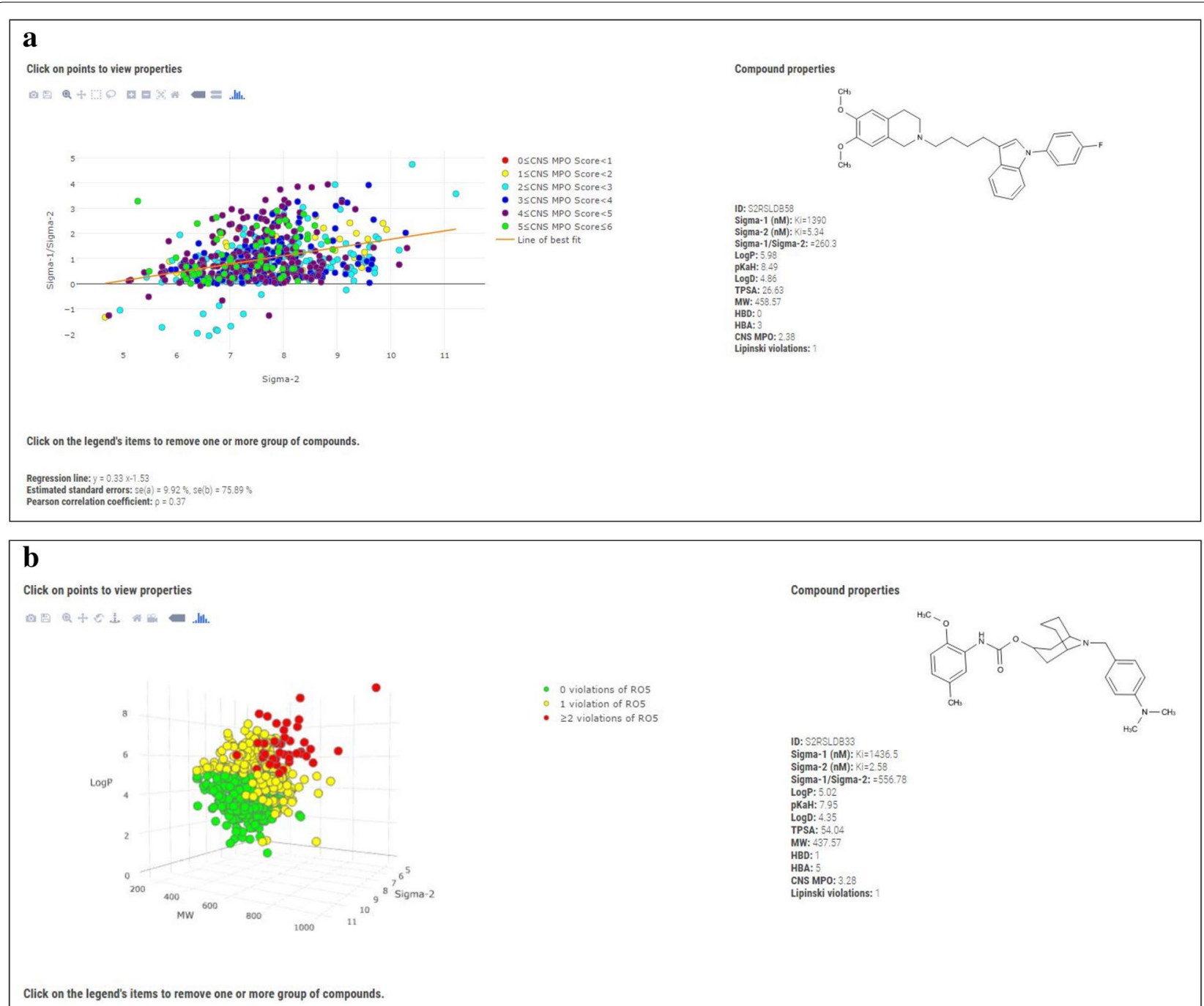

Fig. $42 \mathrm{D}(\mathbf{a})$ and $3 \mathrm{D}(\mathbf{b})$ scatter distribution (website screenshots) of compound's $\sigma_{2} K_{\mathrm{i}}$ versus $\sigma_{1} / \sigma_{2}$ ratio and of compound's $\sigma_{2}$ p $K_{\mathrm{i}}$ versus MW versus logP color coded by CNS MPO score (2D) and Lipinski's rule of five filter (3D), and two compounds together with their property summaries

properties composing the score normalized according to the CNS MPO algorithms. Each compound may thus be opened as a summary page (Fig. 3c).

Similar filtering options are available for the pharmacological properties search. This search function also includes which type of standard constant to be displayed $\left(\mathrm{IC}_{50}, K_{\mathrm{i}}\right.$, and \% of inhibition) for both receptors and the $\sigma_{1} / \sigma_{2}$ ratio (i.e. selectivity). The reference filter search function allows the end user to customize results based on journal name, article title, author name, volume, issue, page and year of publication. Finally, to help the user in retrieving a specific compound from the database S2RSLDB-ID and the reference article compound key and/or preferred name (as reported in the literature source) searches have been embedded.
The user may build complex queries performing searches on a number of fields simultaneously. All the numerical fields in the tabulated results webpage can be sorted, allowing for an enhanced data analysis. Tabulated result page sorting is accomplished with Sorttable (v2) [57]. Compounds can be selected for download in excel binary file format (xls). All the entries in the page are selected by default and a check-all button lets the user select/deselect and then download all the compounds displayed. Navigating in the download tab, the full database may be downloaded in two different file formats: structure-data file (sdf) and xls.

Of particular importance for the data set analysis is the opportunity to perform graphical analysis of the data set or subset. The user may build customized 2D and 3D 
scatter plots of the compounds selected from the tabulated results page, by defining the axes (eleven variables available: Sigma-1 $K_{\mathrm{i}}$, sigma-2 $K_{\mathrm{i}}$, sigma-1/Sigma-2 $K_{\mathrm{i}}$ ratio, $\log P, \mathrm{p} K_{\mathrm{aH}}, \log D$, TPSA, MW, HBD, HBA, and CNS MPO) that in turn will compose the Cartesian coordinates of the $2 \mathrm{D}$ or $3 \mathrm{D}$ graph. A webpage will give the scatter plot for the defined axes. A simple linear regression model has also been added to the 2D graph and this feature should let the user to recognize pattern in the compound properties behaviour. Being $\mathrm{x}$ and $\mathrm{y}$ the two variables to be plotted, the fitted regression line has the following equation: $y=a x+b$, where $a$ is the slope and $b$ is the intercept of the estimated line. Therefore we estimated the standard error (se) for a and b, indicated on the database $2 \mathrm{D}$ plot webpage with se(a) and se(b). Finally, the Pearson correlation coefficient $\rho$ is given in order to appreciate the data fitting [58]. The aforementioned functionalities are run with background software. The plots dots may also be color coded based on Lipinski's rule of five violations or CNS MPO score range. A three or six color code can been applied as a function of the Lipinski's rule of five violations or CNS MPO score range, respectively. Moreover, in the $2 \mathrm{D}$ and $3 \mathrm{D}$ plot webpage, an interactive box has been embedded and this feature allows the user to remove from the plot group of compounds with specific Lipinski's rule of five violations or CNS MPO score range. The graphs are interactive and moving the cursor over a point, will show the coordinates as a tooltip, whilst by clicking a point of the graph, the $2 \mathrm{D}$ image of the selected compound and a properties summary will be returned in a quadrant. The plotting is made available by plotly.js (v1.10.2) [59] which is embedded in the website. Since the pharmacological properties (experimental radioligand binding assay) are variables with a wide numerical distribution, for plotting purposes, we have reported them as $-\log$ of the $K_{\mathrm{i}}$ or $\mathrm{IC}_{50}$ (i.e. as $\mathrm{p} K_{\mathrm{i}}$ or $\left.\mathrm{pIC}_{50}\right)$. For the same reason, the $\sigma_{1} / \sigma_{2}$ ratio is showed in logarithm scale $\left(\log \sigma_{1} / \sigma_{2}\right.$ ratio). This last function may be used prospectively at the design stage to accelerate the identification of $\sigma_{2}$ receptor ligands with increased probability of selectivity over $\sigma_{1}$ receptor. The plots may be downloaded as image files for further offline purposes. Figure 4 shows the 2D (a) and 3D (b) scatter distribution (website screenshots) of compound's $\sigma_{2} K_{\mathrm{i}}$ versus $\sigma_{1} / \sigma_{2}$ ratio and of compound's $\sigma_{2} \mathrm{p} K_{\mathrm{i}}$ versus MW versus $\log \mathrm{P}$ color coded by CNS MPO score (2D) and Lipinski's rule of five filter (3D), and two compounds together with their property summaries.

\section{Conclusion}

The $\sigma_{2}$ receptor structure has not yet been disclosed, and radioligand binding assay is primarily used to understand the receptor's pharmacological behavior and design new lead compounds. With this in mind, here we present a comprehensive, manually curated, database of the $\sigma_{2}$ receptor selective ligands containing more than 650 compounds, built with chemical structure information, radioligand binding affinity data, computed physicochemical properties, and experimental binding protocols. The reported data have been manually retrieved from the literature thus keeping this database highly reliable. Each compound in the database has the reference source. The S2RSLDB is freely available online without account login and having a powerful search engine the user may build complex queries, sort tabulated results, generate 2D and 3D color coded graphs and download the data for additional offline screening. The collection here reported is extremely useful for the development of new ligands endowed of $\sigma_{2}$ receptor affinity, selectivity, and appropriate physicochemical properties.

\section{Abbreviations}

CNS MPO: central nervous system multiparameter optimization; TPSA: topological polar surface area; MW: molecular weight; PET: positron emission tomography; SMILES: simplified molecular-input line-entry system; SMARTS: SMiles ARbitrary target specification; InChl: IUPAC international chemical identifier; HTML: hypertext markup language; CSS: cascading style sheets; JSME: JavaScript molecule editor; 2D: two-dimensional; 3D: three-dimensional; S2RSLDB: sigma-2 receptor selective ligands database.

\section{Authors' contributions}

EA conceived and designed S2RSLDB. GN developed the database and GUI infrastructure. CM data analysis, data collection and supporting the database development. CM, VP, MNM, OP, GR, AR, AM and EA experimental data and interpretations. GN, AM and AR helped to draft the manuscript. EA drafted the manuscript and supervised database and GUI infrastructure development. All authors read and approved the final manuscript.

\section{Acknowledgements}

The authors are thankful to Mr. Dario Molè for his helpful contribute in website graphic design and to Dr. Gerardo Maiorano for his support with server handling. Free academic license from ChemAxon and OpenEye Scientific Software for their suites of programs is gratefully acknowledged.

\section{Competing interests}

The authors declare that they have no competing interests.

\section{Funding}

This work was supported by Research Funding for University (FIR) 2014.

Received: 6 June 2016 Accepted: 16 January 2017

Published online: 21 January 2017

\section{References}

1. Quirion R, Bowen WD, Itzhak Y, Junien JL, Musacchio JM, Rothman RB, Su TP, Tam SW, Taylor DP (1992) A proposal for the classification of sigma binding sites. Trends Pharmacol Sci 13(3):85-86

2. Matsumoto RR, Bowen WD, Su TP (2007) Sigma receptors, chemistry, cell biology and clinical implications. Springer, New York

3. Hanner M, Moebius FF, Flandorfer A, Knaus HG, Striessnig J, Kempner E, Glossmann H (1996) Purification, molecular cloning, and expression of the mammalian sigma1-binding site. Proc Natl Acad Sci USA 93(15):8072-8077 
4. Hayashi T, Su TP (2007) Sigma-1 receptor chaperones at the ER-mitochondrion interface regulate $\mathrm{Ca}(2+)$ signaling and cell survival. Cell 131(3):596-610

5. Kekuda R, Prasad PD, Fei YJ, Leibach FH, Ganapathy V (1996) Cloning and functional expression of the human type 1 sigma receptor (hSigmaR1). Biochem Biophys Res Commun 229(2):553-558

6. Schmidt HR, Zheng S, Gurpinar E, Koehl A, Manglik A, Kruse AC (2016) Crystal structure of the human sigma1 receptor. Nature 532(7600):527-530

7. Chu UB, Ruoho AE (2016) Biochemical pharmacology of the sigma-1 receptor. Mol Pharmacol 89(1):142-153

8. Guo L, Zhen X (2015) Sigma-2 receptor ligands: neurobiological effects. Curr Med Chem 22(8):989-1003

9. Hellewell SB, Bruce A, Feinstein G, Orringer J, Williams W, Bowen WD (1994) Rat liver and kidney contain high densities of sigma 1 and sigma 2 receptors: characterization by ligand binding and photoaffinity labeling. Eur J Pharmacol 268(1):9-18

10. Chu UB, Mavlyutov TA, Chu ML, Yang H, Schulman A, Mesangeau C, McCurdy CR, Guo LW, Ruoho AE (2015) The sigma-2 receptor and progesterone receptor membrane component 1 are different binding sites derived from independent genes. EBioMedicine 2(11):1806-1813

11. Xu J, Zeng C, Chu W, Pan F, Rothfuss JM, Zhang F, Tu Z, Zhou D, Zeng D, Vangveravong S, Johnston F, Spitzer D, Chang KC, Hotchkiss RS, Hawkins WG, Wheeler KT, Mach RH (2011) Identification of the PGRMC1 protein complex as the putative sigma-2 receptor binding site. Nat Commun 2:380

12. Prezzavento $O$, Campisi A, Parenti $C$, Ronsisvalle $S$, Arico G, Arena E, Pistolozzi M, Scoto GM, Bertucci C, Vanella A, Ronsisvalle G (2010) Synthesis and resolution of cis- $( \pm)$-methyl (1R,2S/1S,2R)-2-[(4-hydroxy-4-phenylpiperidin-1-yl)methyl]-1-(4-methylphenyl)cycl opropanecarboxylate [( \pm )-PPCC)]: new sigma receptor ligands with neuroprotective effect. J Med Chem 53(15):5881-5885

13. Maurice $T$ (2002) Improving Alzheimer's disease-related cognitive deficits with sigma1 receptor agonists. Drug News Perspect 15(10):617-625

14. Hayashi T (2015) Sigma-1 receptor: the novel intracellular target of neuropsychotherapeutic drugs. J Pharmacol Sci 127(1):2-5

15. Prezzavento O, Arena E, Sanchez-Fernandez C, Turnaturi R, Parenti C, Marrazzo A, Catalano R, Amata E, Pasquinucci L, Cobos EJ (2017) (+)-and (-)-Phenazocine enantiomers: evaluation of their dual opioid agonist/ sigma1 antagonist properties and antinociceptive effects. Eur J Med Chem 125:603-610

16. Olivieri M, Amata E, Vinciguerra S, Fiorito J, Giurdanella G, Drago F, Caporarello N, Prezzavento O, Arena E, Salerno L, Rescifina A, Lupo G, Anfuso CD, Marrazzo A (2016) Antiangiogenic Effect of ( \pm )-haloperidol metabolite ii valproate ester [( \pm )-MRJF22] in human microvascular retinal endothelial cells. J Med Chem 59(21):9960-9966

17. Davis MP (2015) Sigma-1 receptors and animal studies centered on pain and analgesia. Expert Opin Drug Discov 10(8):885-900

18. Hayashi T, Rizzuto R, Hajnoczky G, Su TP (2009) MAM: more than just a housekeeper. Trends Cell Biol 19(2):81-88

19. Prezzavento O, Arena E, Parenti C, Pasquinucci L, Arico G, Scoto GM Grancara S, Toninello A, Ronsisvalle S (2013) Design and synthesis of new bifunctional sigma-1 selective ligands with antioxidant activity. J Med Chem 56(6):2447-2455

20. Weatherspoon JK, Gonzalez-Alvear GM, Frank AR, Werling LL (1996) Regulation of $[3 \mathrm{H}]$ dopamine release from mesolimbic and mesocortical areas of guinea pig brain by sigma receptors. Schizophr Res 21(1):51-62

21. Maurice T, Su TP (2009) The pharmacology of sigma-1 receptors. Pharmacol Ther 124(2):195-206

22. Sanchez C, Arnt J, Costall B, Kelly ME, Meier E, Naylor RJ, Perregaard J (1997) The selective sigma2-ligand Lu 28-179 has potent anxiolytic-like effects in rodents. J Pharmacol Exp Ther 283(3):1323-1332

23. Mach RH, Zeng C, Hawkins WG (2013) The sigma2 receptor: a novel protein for the imaging and treatment of cancer. J Med Chem 56(18):7137-7160

24. Ostenfeld MS, Fehrenbacher N, Hoyer-Hansen M, Thomsen C, Farkas T, Jaattela M (2005) Effective tumor cell death by sigma-2 receptor ligand siramesine involves lysosomal leakage and oxidative stress. Cancer Res 65(19):8975-8983

25. Ronsisvalle S, Arico G, Cova AM, Blanco P, Amata E, Pappalardo M, Pasquinucci L, Spadaro A, Ronsisvalle N (2016) Caspase-3 activation in human melanoma A375 cell line by a novel selective sigma-2 agonist. Pharmazie 71(3):146-151

26. Bem WT, Thomas GE, Mamone JY, Homan SM, Levy BK, Johnson FE, Coscia CJ (1991) Overexpression of sigma receptors in nonneural human tumors. Cancer Res 51(24):6558-6562

27. Crawford KW, Bowen WD (2002) Sigma-2 receptor agonists activate a novel apoptotic pathway and potentiate antineoplastic drugs in breast tumor cell lines. Cancer Res 62(1):313-322

28. Mach RH, Smith CR, Al-Nabulsi I, Whirrett BR, Childers SR, Wheeler KT (1997) Sigma 2 receptors as potential biomarkers of proliferation in breast cancer. Cancer Res 57(1):156-161

29. Al-Nabulsi I, Mach RH, Wang LM, Wallen CA, Keng PC, Sten K, Childers SR, Wheeler KT (1999) Effect of ploidy, recruitment, environmental factors, and tamoxifen treatment on the expression of sigma-2 receptors in proliferating and quiescent tumour cells. Br J Cancer 81(6):925-933

30. Schinina B, Martorana A, Colabufo NA, Contino M, Niso M, Perrone MG, De Guidi G, Catalfo A, Rappazzo G, Zuccarello E, Prezzavento O, Amata E, Rescifina A, Marrazzo A (2015) 4-nitro-2,1,3-benzoxadiazole derivatives as potential fluorescent sigma receptor probes. RSC Adv 5(58):47108-47116

31. Srinivasarao M, Galliford CV, Low PS (2015) Principles in the design of ligand-targeted cancer therapeutics and imaging agents. Nat Rev Drug Discov 14(3):203-219

32. Makvandi M, Lieberman BP, LeGeyt B, Hou C, Mankoff DA, Mach RH, Pryma DA (2016) The pre-clinical characterization of an alpha-emitting sigma-2 receptor targeted radiotherapeutic. Nucl Med Biol 43(1):35-41

33. Washington University School of Medicine: PET Assessment of Acute Lung Transplant Rejection. In: ClinicalTrials.gov. https://clinicaltrials.gov/ show/NCT02204202. NLM Identifier: NCT02204202

34. Abramson Cancer Center of the University of Pennsylvania: Imaging of In Vivo sigma-2 Receptor Expression With $\left[{ }^{18} \mathrm{~F}\right] \mathrm{ISO}-1$ Positron Emission Tomography (PET/CT) in Primary Breast Cancer. In: ClinicalTrials. gov. https://clinicaltrials.gov/ct2/show/NCT02762110. NLM Identifier: NCT02762110

35. Washington University School of Medicine: $\left[{ }^{18} \mathrm{~F}\right] \mathrm{ISO}-1$ PET/CT in Breast Cancer. In: ClinicalTrials.gov. https://clinicaltrials.gov/ct2/show/ NCT02284919. NLM Identifier: NCT02284919

36. Washington University School of Medicine: Assessment of Cellular Proliferation in Tumors by Positron Emission Tomography (PET) Using $\left[{ }^{18} \mathrm{~F}\right] \mathrm{ISO}-1$ (FISO PET/CT). In: ClinicalTrials.gov. https://clinicaltrials.gov/ct2/ show/NCT00968656. NLM Identifier: NCT00968656

37. Pubmed. http://www.ncbi.nlm.nih.gov/pubmed

38. Scifinder. https://www.cas.org/products/scifinder

39. Binding Database. https://www.bindingdb.org

40. ChEMBL_21. https://www.ebi.ac.uk/chembl

41. PubChem. https://pubchem.ncbi.nlm.nih.gov

42. PDBbinding. http://www.pdbbind.org

43. ChemSpider. www.chemspider.com

44. ChemAxon-cheminformatics platforms and desktop applications. https://www.chemaxon.com/

45. Filippov IV, Nicklaus MC (2009) Optical structure recognition software to recover chemical information: OSRA, an open source solution. J Chem Inf Model 49(3):740-743

46. O'Boyle NM, Banck M, James CA, Morley C, Vandermeersch T, Hutchison GR (2011) Open Babel: an open chemical toolbox. J Cheminform 3:33

47. Chu UB, Ruoho AE (2015) Sigma receptor binding assays. Curr Protoc Pharmacol 71(1.34):1-21

48. O'Boyle NM, Morley C, Hutchison GR (2008) Pybel: a python wrapper for the OpenBabel cheminformatics toolkit. Chem Cent J 2:5

49. Bienfait B, Ertl P (2013) JSME: a free molecule editor in JavaScript. J Cheminform 5:24

50. Indigo Toolkit. http://lifescience.opensource.epam.com/

51. Bajusz D, Racz A, Heberger K (2015) Why is Tanimoto index an appropriate choice for fingerprint-based similarity calculations? J Cheminform 7:20

52. Ehrlich HC, Rarey M (2012) Systematic benchmark of substructure search in molecular graphs - From Ullmann to VF2. J Cheminform 4(1):13

53. Heller S, McNaught A, Stein S, Tchekhovskoi D, Pletnev I (2013) InChl-the worldwide chemical structure identifier standard. J Cheminform 5(1):1-9

54. Lipinski CA, Lombardo F, Dominy BW, Feeney PJ (2001) Experimental and computational approaches to estimate solubility and permeability in drug discovery and development settings. Adv Drug Deliv Rev 46(1-3):3-26 
55. Wager TT, Hou X, Verhoest PR, Villalobos A (2010) Moving beyond rules: the development of a central nervous system multiparameter optimization (CNS MPO) approach to enable alignment of druglike properties. ACS Chem Neurosci 1(6):435-449

56. Diaz R, Luengo-Arratta SA, Seixas JD, Amata E, Devine W, Cordon-Obras C, Rojas-Barros DI, Jimenez E, Ortega F, Crouch S, Colmenarejo G, Fiandor JM, Martin JJ, Berlanga M, Gonzalez S, Manzano P, Navarro M, Pollastri MP (2014) Identification and characterization of hundreds of potent and selective inhibitors of Trypanosoma brucei growth from a kinase-targeted library screening campaign. PLoS Negl Trop Dis 8(10):e3253
57. Sorttable: Make all your tables sortable. http://www.kryogenix.org/code/ browser/sorttable/

58. Montgomery DC, Peck EA, Vining GG (2013) Applied statistics and probability for engineers, 6 th edn. Wiley, Hoboken

59. Plotly.js - a JavaScript graphing library, open-sourced. https://plot.ly/ javascript/

\section{Submit your manuscript to a SpringerOpen ${ }^{0}$ journal and benefit from:}

- Convenient online submission

- Rigorous peer review

- Immediate publication on acceptance

- Open access: articles freely available online

- High visibility within the field

- Retaining the copyright to your article 